\title{
GPPS-BJ-2019-0025
}

\section{Control of the axial compressor blade airfoil boundary layer flow using plasma actuation}

\author{
Haideng Zhang ${ }^{1,2}$ \\ 1. Key Laboratory on Plasma Dynamics \\ 2. Xi'an Jiaotong University \\ zhanghaideng@126.com \\ Xi'an, China
}

\author{
Yun $\mathrm{Wu}^{1,2}$ \\ 1. Key Laboratory on Plasma Dynamics \\ 2. Xi'an Jiaotong University \\ wuyun1223@126.com \\ Xi'an, China
}

\author{
Yinghong Li \\ Key Laboratory on Plasma Dynamics \\ yinghong1i@163.com \\ Xi’an, China
}

\begin{abstract}
The control effect of plasma actuation on the boundary layer flow of a typical CDA compressor airfoil is investigated in this paper using large scale simulation method. The reliability and accuracy of the simulation method is validated firstly. Then the characteristics of the compressor airfoil boundary layer flow as well as the flow control effects of the plasma actuation located at the blade leading edge on the compressor airfoil boundary layer flow are investigated. The plasma actuations located at the blade leading edge can induce a small spanwise vortex through influencing on the density and pressure of the flow field. As the small spanwise vortex moves downstream along the blade surface with the main flow, it can suppress the turbulent flow mixing and reduce the total pressure loss. The flow control effect of the small spanwise vortex is summarized as wall jet effect. However, the small spanwise vortex induced by the plasma actuation will increase the total pressure loss of the laminar boundary layer flow while moving by.
\end{abstract}

\section{INTRODUCTION}

Boundary layer flow is very crucial to the aerodynamic performance of the compressor airfoil. It is well known that the appearance of CDA (controlled diffusion airfoil) improves the aerodynamic performance of axial compressor dramatically, and the advantage of CDA is rooted in that the compressor airfoil boundary layer flow is well controlled through adjusting the distribution of adverse pressure gradient (Hobbs and Weingold, 1984). Although it is possible to achieve the perfect boundary layer flow state through optimizing the aerodynamic design, the abrasion of the blade during usage as well as the machining error will deteriorate the boundary layer flow, especially at off-design conditions (Goodhand, 2011). So it is of great significance to develop the active control technique to control the compressor airfoil flow.

Plasma actuation is a new-type active flow control technique, and is characterized by simple structure and wide actuation frequency band (Cork et al., 2010). So it is advantageous to use plasma actuation to control the boundary layer flow. Based on the wave form of the actuation voltage, the dielectric barrier discharge plasma actuation can be classified into AC-DBD and NS-DBD plasma actuation. The AC-DBD plasma actuation controls the flow mainly through inducing body force in the flow field, and the NS-DBD plasma actuation controls the flow mainly through inducing strong disturbance in the flow field.

Kriegseis et al. found that the AC-DBD plasma actuation can delay the boundary layer transition of aircraft wing to reduce the friction loss and resistance (Kriegseis et al., 2016). Zheng et al. found that the NS-DBD plasma actuation can control the boundary layer flow separation effectively through inducing spanwise vortex structures (Zheng et al., 2019). These vortex structures can interact with the boundary layer flow and suppress the flow separations. Zhang et al. found that the AC-DBD plasma actuation can suppress the compressor corner separation and tip leakage flow (Zhang et al., 2017; Zhang et al., 2019). The control effect of the plasma actuation on the compressor flow has been investigated by other researchers too (Akcayoz et al., 2016; De Giorgi et al., 2016; Li et al., 2010; Saddoughi et al., 2016; Vo et al., 2018; Vo et al., 2012; Yu et al., 2019; Saddoughi et al., 2014; Vo, 2010; Vo et al., 2008; Wadia et al., 2012). However, the control effect of plasma actuation on the turbomachinery boundary layer flow remain uncovered. 
To develop the active control technique which can control the compressor airfoil boundary layer flow effectively, this paper investigates the control effect of NS-DBD plasma actuation on the boundary layer flow of a typical CDA airfoil. The results obtained can further guide the design of active control technique of compressor airfoil boundary layer flow.

\section{SIMULATION METHOD}

A typical CDA airfoil is studied in this paper. The airfoil model as well as the corresponding main aerodynamic parameters are shown in Fig. 1.

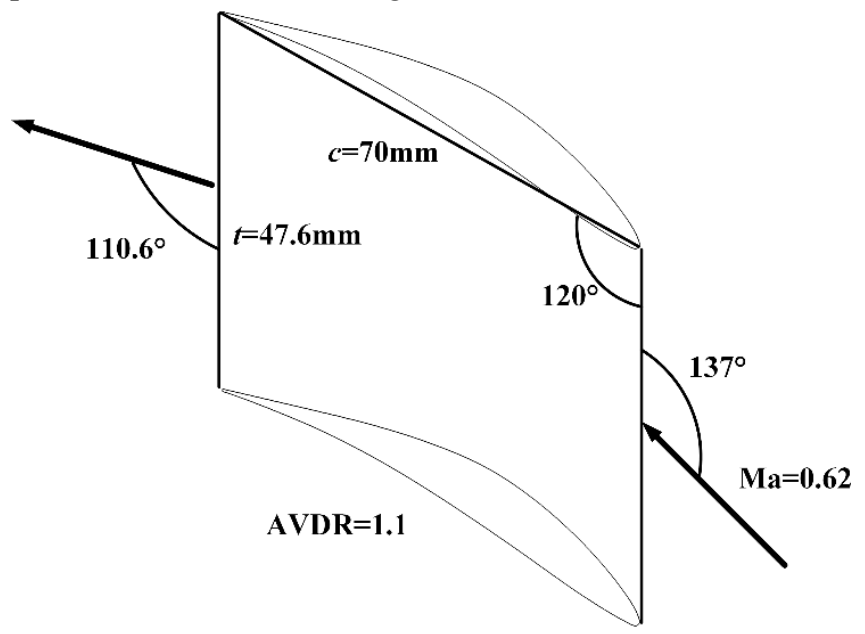

Fig. 1 The CDA airfoil model

The Numeca/IGG software is used to establish the simulation grid, and the topological structure is $\mathrm{HOH}$. To meet the requirement of LES simulations, the grid is refined to ensure the non-dimensional grid size in spanwise, streamwise and tangential directions no bigger than 50 . The total number of the simulation grid is 4.5 million and the LES Wale model is used to simulate the flow field. While the plasma actuation is imposed in the flow field, the simulation time step is 5 nanosecond, and the simulation time step is changed to 50 nanosecond after the plasma actuation is imposed to accelerate the simulation procedure. It takes about 6000 time steps, which corresponds to 0.0003 second, for the fluid to move from the blade leading edge to the trailing edge.

The distributions of isentropic Mach number at design incidence angle from both simulation and experiment(Steinert et al., 1990) are shown in Fig. 2. It can be found that the compressor airfoil flow is well predicted by the simulation method used in this paper.

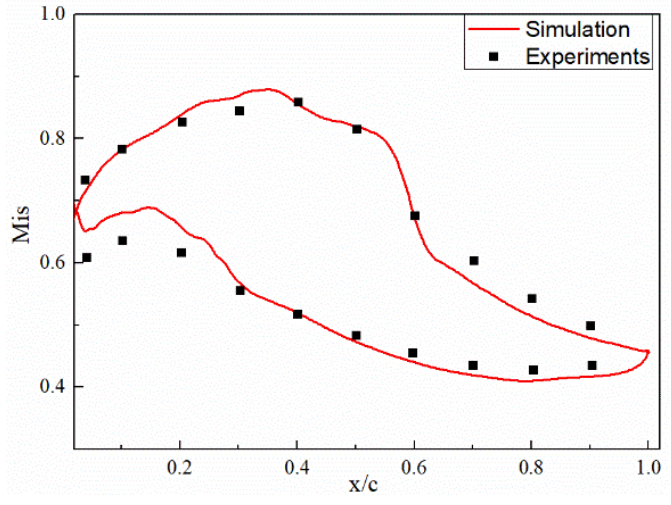

Fig. 2 Comparisons between simulation and experiment

\section{PLASMA ACTUATION MODEL}

The plasma actuation is simulated using the phonological model and imposed on the blade surface. The temperature distribution after the plasm actuation is imposed is shown in Fig. 3. It can be found that since the energy is injected into the flow field in a very short time interval (50 nanosecond), the local temperature is increased dramatically. As a result, the compressive wave as well as spanwise vortex structure will be aroused. These flow structures will interact with the blade boundary layer flow and the flow control effect is expected to be achieved.

During the simulations, the total energy input as well as the actuation location is varied to investigate the influences of plasma actuation strength on the flow control effects.

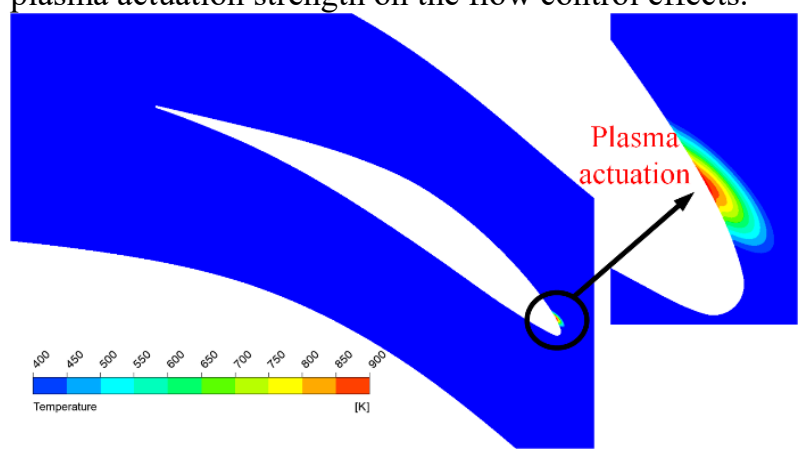

Fig. 3 Temperature distribution after the plasma actuation is imposed.

To verify the reliability of the plasma actuation model, the influences of the nanosecond plasma actuation on the static flow field are investigated and compared to related experimental results. During the simulations, the plasma actuation region can be represented by the temperature distributions since the influence of the plasma actuation is treated as fast heating. Then the plasma actuation region obtained from simulation and experiments is shown in Fig. 4, where the plasma actuation region is experimentally measured based on plasma emission(Aleksandrov et al., 2010). It can be found that the shape of plasma actuation region is qualitatively captured with the plasma actuation model. 


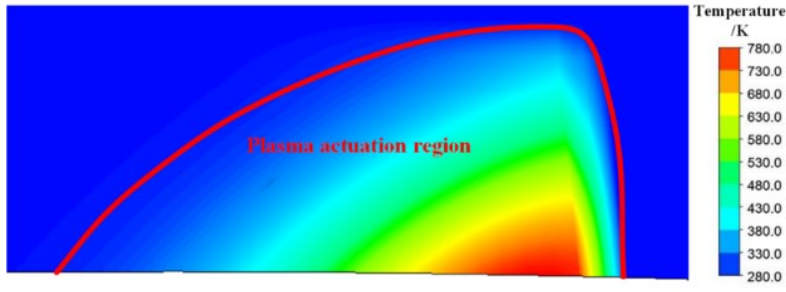

(a) Simulation

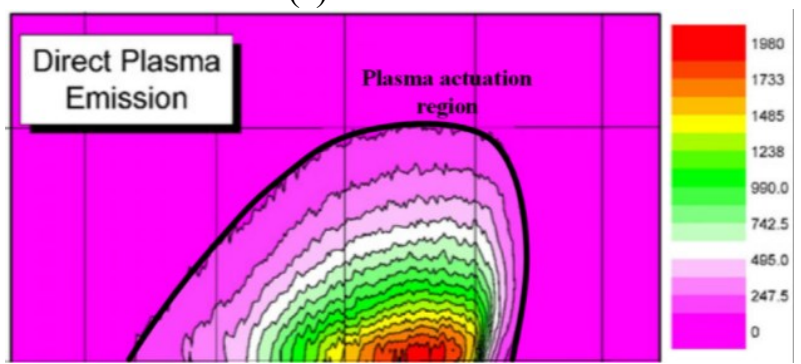

(b) Experiment(Aleksandrov et al., 2010)

Fig. 4 The obtained plasma actuation region.

It is well known that the nanosecond plasma actuation can induce compressive wave through imparting fast heating into the flow field, and the compressive wave simulated with the plasma actuation model is shown in Fig. 5(a). With time going by, the compressive wave will spread outwards from the plasma actuation region to the flow field, and the positions of the compressive wave along the SL line (shown in Fig. 5 (a)) from both simulation and experiment(Starikovskii et al., 2009) are shown in in Fig. 5 (b). It can be found that the development of the compressive wave is well captured with the plasma actuation model. With the results of Fig. 4 and Fig. 5 , it can be concluded that the plasma actuation model used in this paper can capture the influences of plasma actuation on the flow field successfully.

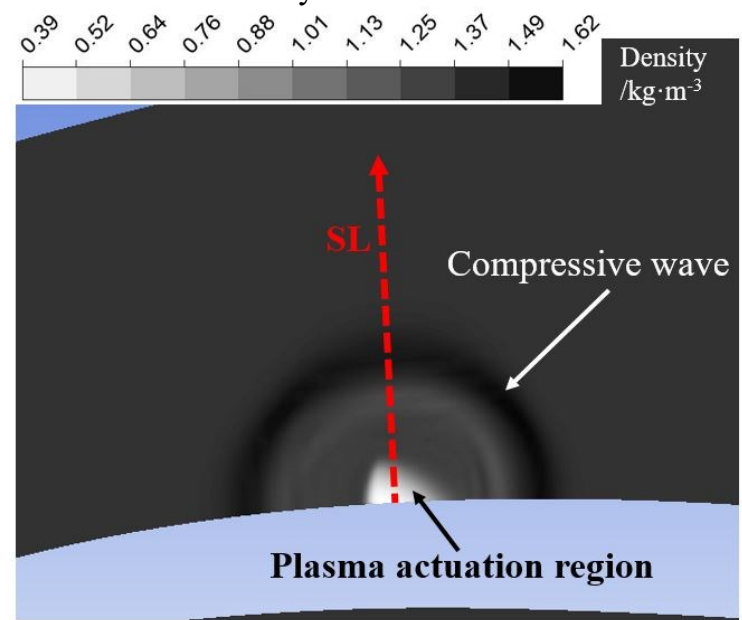

(a) Compressive wave

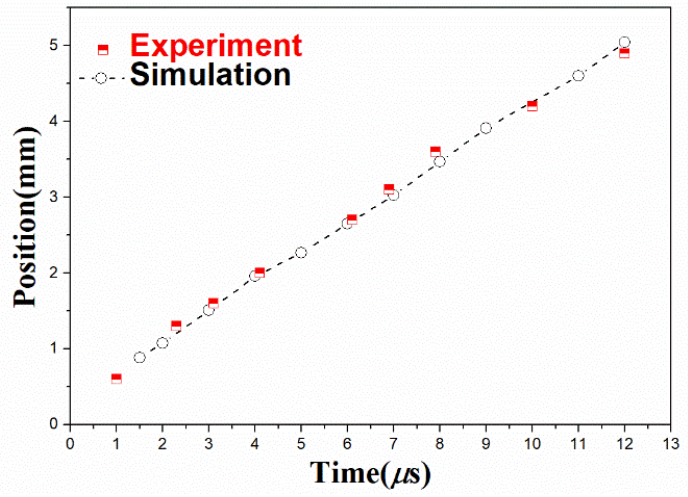

(b) Development of the compressive wave

Fig. 5 Characteristics of the compressive wave induced by plasma actuation

\section{CHARACTERISTICS OF THE COMPRESSOR AIRFOIL BOUNDARY LAYER FLOW}

The time-averaged static pressure and axial wall shear stress coefficients on the blade suction surface are shown in Fig. 6. The static pressure coefficient $C_{p}$ is defined as

$$
C_{p}=\left(\bar{p}-\bar{p}_{1}\right) / \frac{1}{2} \rho U_{1}^{2}
$$

where $\bar{p}$ is the time-averaged local static pressure and $\overline{p_{1}}$ is the time-averaged static pressure of inlet flow. $U_{1}$ is the timeaveraged velocity of inlet flow. As for the simulation cases without plasma actuation, the time-averaged results here and in the following parts are obtained from arithmetical average of transient results from 18 thousand time steps during which the fluid is expected to flow pass the blade passage three times. Similarly, the axial wall shear stress coefficient $C_{f}$ is defined as

$$
C_{f}=\bar{\tau}_{a} / \frac{1}{2} \rho U_{1}^{2}
$$

where $\overline{\tau_{a}}$ is the time-averaged axial wall shear stress on the blade surface.

According to Fig. 6, there exists a T-zone on the blade suction surface. Within the T-zone, the static pressure increases quickly and the axial wall shear stress becomes negative, which indicate the occurrence of boundary layer transition and reversal flow. Since the reversal flow is related to flow separation, the boundary layer transition within the Tzone is triggered by laminar separation bubble. These findings are in accordance with previous experimental results, where boundary layer transition and laminar separation bubble are observed from experimental measurements(Steinert and Starken, 1996). 


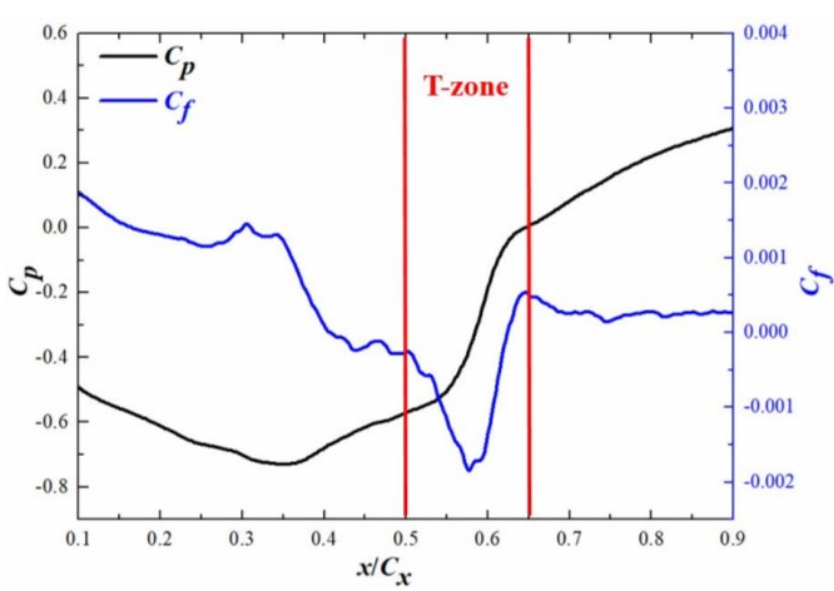

Fig. 6 Time-averaged static pressure and axial wall shear stress coefficients on the blade suction surface: black line, $\mathrm{Cp}$ and blue line, $\mathrm{Cf}$

The contour of the time-averaged total pressure loss coefficient on the mid-span S1 surface is shown in Fig. 7. The time-averaged total pressure loss coefficient $\omega$ is defined as

$$
\omega=\left(\bar{p}_{t 1}-\bar{p}_{t}\right) / \frac{1}{2} \rho U_{1}^{2}
$$

where $\overline{p_{t 1}}$ and $\overline{p_{t}}$ are the time-averaged total pressure of inlet flow and local flow respectively. It can be found that apart from the blade wake, there exists a high total pressure loss region near the blade suction surface within the T-zone. Based on the streamlines of the high total pressure loss region, the laminar separation bubble which triggers boundary layer transition can be observed.

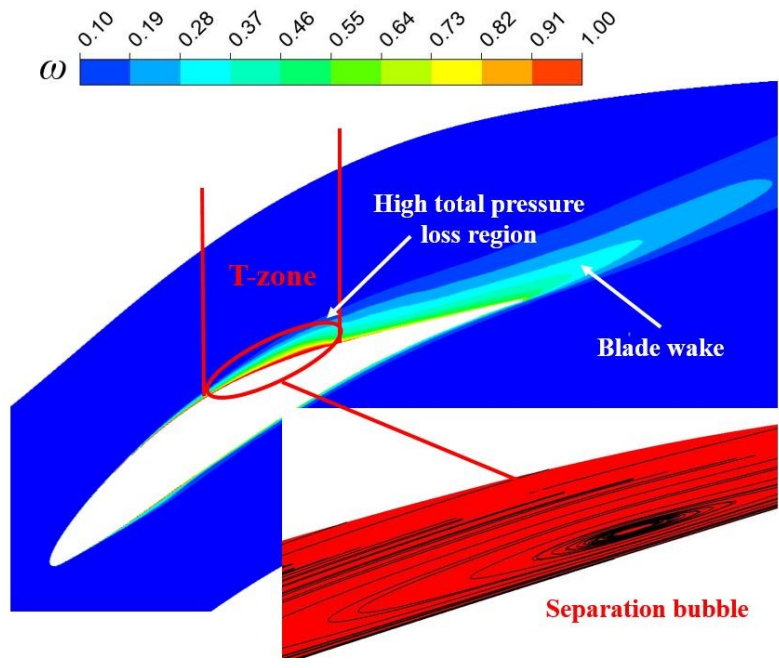

Fig. 7 Contour of the time-averaged total pressure loss coefficient on the $\mathrm{S} 1$ surface.

Transient flow structures around the blade by iso-surfaces of Q criterion are shown in Fig. 8, and the flow structures are colored by time-averaged total pressure loss coefficient. It can be found that the boundary layer flow remains as laminar at the front part of the blade, and transits to turbulent at the rear part of the blade. The laminar separation bubble can be observed within the boundary layer transition region. As flow reattaches after flowing pass the separation bubble, the boundary layer flow becomes complete turbulent. Although large-scale turbulent flow structures can be observed at the rear part of the blade, the high total pressure loss region is limited to the near wall flow region, especially within the laminar separation bubble.

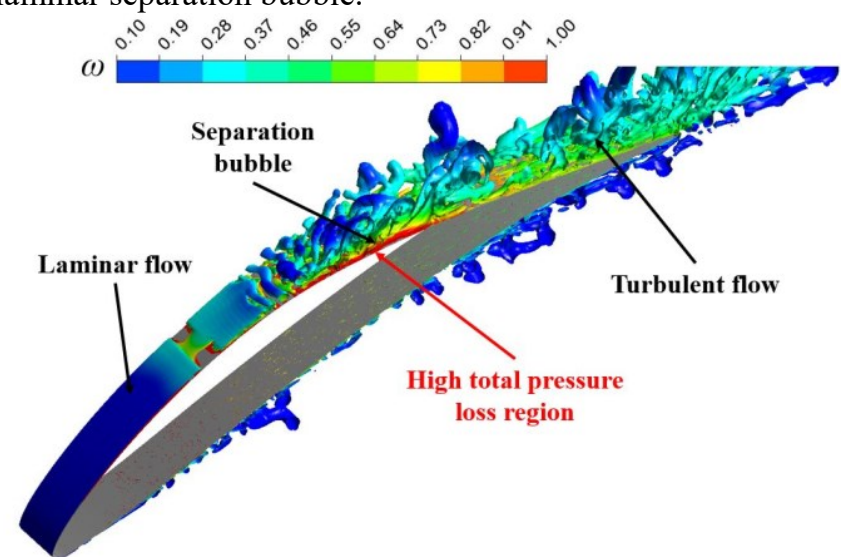

Fig. 8 Transient flow structures around the blade by isosurfaces of Q criterion, and colored by the total pressure loss coefficient.

\section{FLOW CONTROL EFFECTS OF THE PLASMA ACTUATION ON THE COMPRESSOR AIRFOIL BOUNDARY LAYER FLOW}

The contours of spanwise vorticity $\left(\omega_{\mathrm{s}}\right)$ on the mid-span S1 surface at different moments after Plasma actuation is imposed are shown in Fig. 9. According to Fig. 9(a), the compressive wave can be observed $2 \mu \mathrm{s}$ after the Plasma actuation is imposed. Then the compressive wave will spread outwards and increase the pressure of the flow field. According to Fig. 9(b), a small vortex structure (SVS) with concentrated spanwise vorticity can be observed on the blade suction surface $22.5 \mu$ s after the Plasma actuation is imposed. Then the SVS will move downstream along the blade suction surface with the main flow, and interact with the laminar and turbulent boundary layer flow. According to previous findings, the SVS is mainly caused by the influences of nanosecond plasma actuation on the density and pressure of the flow field(Zhao et al., 2015).

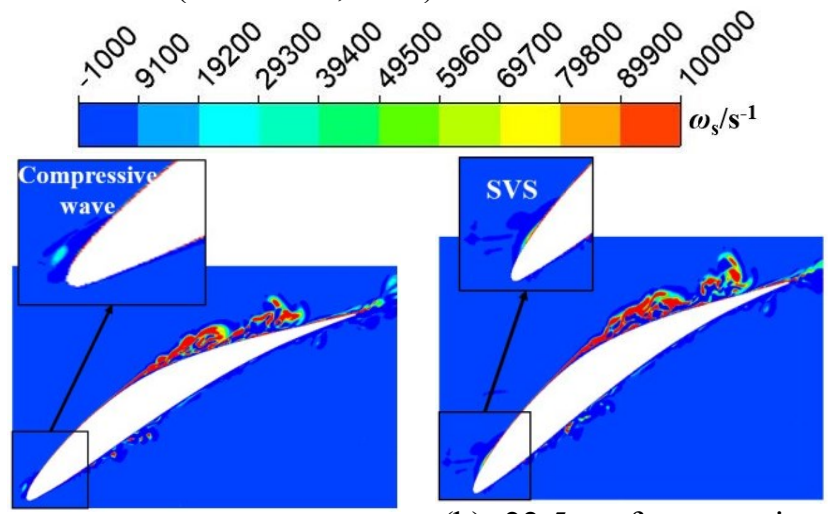

(a) $2 \mu \mathrm{s}$ after actuation

(b) $22.5 \mu \mathrm{s}$ after actuation

Fig. 9 Contours of spanwise vorticity on the mid-span S1 surface at different moments after Plasma actuation is imposed. 
According to the vorticity transport equations, the variance of vorticity can be attributed to the movement of vortex, baroclinic effect of the fluid and the viscosity of the fluid. Researchers have found that the nanosecond plasma actuation can arouse baroclinic effect among the fluid, and induce the spanwise vortex resultantly. The contour of spanwise vorticity around the blade leading edge is shown in Fig. 10 , and the contour of item $\nabla \rho \times \nabla p / \rho^{2}$, which represents the contribution of baroclinic effect to the variance of vorticity, is given too. It can be found that within the SVS region, the baroclinic effect of the fluid is very strong. This indicates the SVS observed in this paper is closely related with the fluid baroclinicity which is caused by the influences of nanosecond plasma actuation on the density and pressure of the flow field.
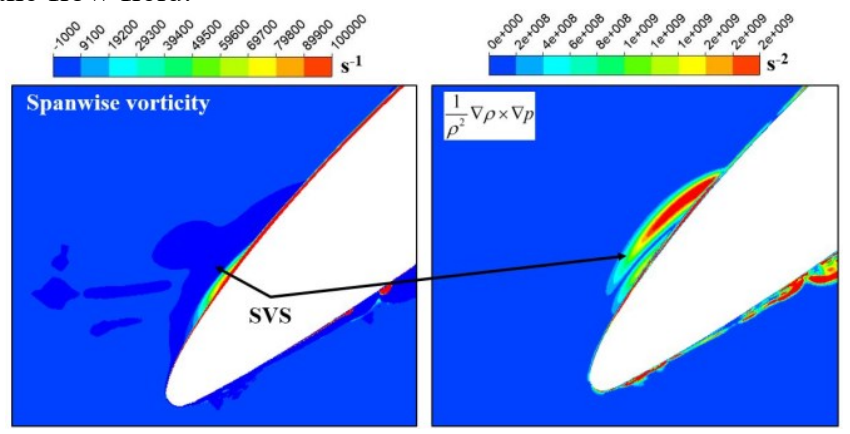

Fig. 10 Contours of spanwise vorticity and item $\nabla \rho \times$ $\nabla p / \rho^{2}$ on the mid-span S1 surface around the blade leading edge $22.5 \mu$ s after plasma actuation is imposed.

As the SVS moves downstream and interacts with the blade suction surface boundary layer flow, the flow state of the boundary layer will be changed. To find out the influences of the SVS on the blade suction surface boundary layer flow, the variance of time-averaged total pressure loss coefficient with and without plasma actuation is defined as $\Delta \omega=\overline{\omega_{a}}-$ $\overline{\omega_{b}}$, where $\overline{\omega_{a}}$ and $\overline{\omega_{b}}$ are the time-averaged total pressure loss coefficient with and without plasma actuation respectively. The contour of $\Delta \omega$ on the mid-span S1 surface with Plasma actuation is shown in Fig. 11. As for the simulation cases with plasma actuation, the time-averaged results here and in the following parts are obtained from arithmetical average of transient results from 40 thousand time steps during which 10 nanosecond plasma actuations are imposed. According to Fig. 11, it can be found that as the SVS moves within the laminar boundary layer flow, the total pressure loss coefficient is increased. The laminar boundary layer flow as well as the main flow is well organized, and the corresponding viscosity flow loss is almost negligible. While moving downstream, the velocity of the SVS is different from the surrounding fluid. As a result, extra flow mixing will be aroused as the SVS interacts with the laminar boundary layer flow as well as the main flow. This is the mechanism behind the increase of total pressure loss coefficient at the front part of the blade. On the contrary, the total pressure loss coefficient is reduced as the SVS moves within the turbulent boundary layer flow. This indicates that the strong flow mixing within the turbulent flow region is suppressed by the movement of the SVS.

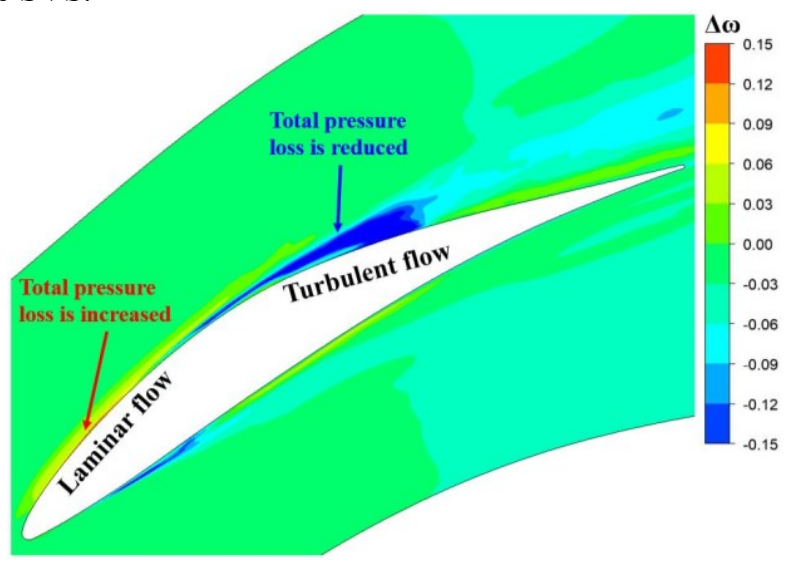

Fig. 11 Contour of the variance of time-averaged total pressure loss coefficient on the S1 surface with Plasma actuation.

The contours of spanwise vorticity with and without plasma actuation on the mid-span S1 surface $149.5 \mu$ s after Actulplasma actuation is imposed are shown in Fig. 12. According to the simulation results, the SVS arrives at the turbulent flow region $74.5 \mu \mathrm{s}$ after Plasma actuation is imposed. Then the SVS continues to move downstream within the turbulent flow region and interacts with the turbulent boundary layer flow. According to Fig. 12, as the SVS moves within the turbulent boundary layer flow region, the spanwise vorticity is reduced which indicates the turbulent flow mixing is suppressed by the movement of SVS. This is the mechanism behind the reduction of total pressure loss coefficient in Fig. 11. In other words, as the SVS moves within the turbulent flow region, it can act as a typical wall jet with streamwise momentum and suppress the strong flow mixing, which will result in the reduction of total pressure loss. The influences of the Plasma actuation on the compressor airfoil boundary layer flow can be observed more clearly from the short movie consists of simulation results at different moments, and the movie has been provided as the supplementary material of this paper.

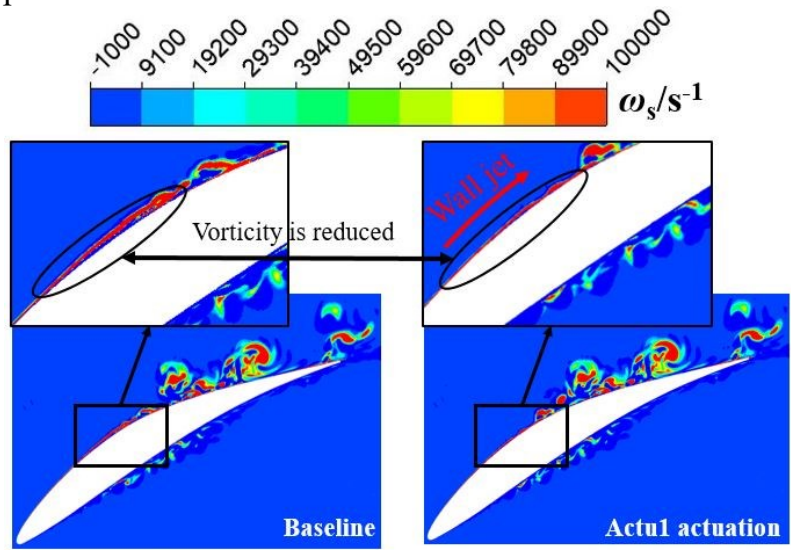

Fig. 12 Contours of spanwise vorticity on the mid-span S1 surface $149.5 \mu$ s after Plasma actuation is imposed (Left is the result without plasma actuation and right is the result with Plasma actuation). 
The influences of plasma actuation energy input are shown in Fig. 13. It can be found that as the energy input per pulse reduces to $0.4 \mathrm{~mJ}$, the plasma actuation can still suppress the boundary layer vortex structures. This indicates that the compressor boundary layer flow can be controlled using plasma actuation with little energy input.

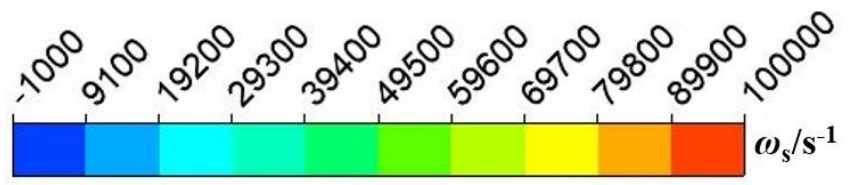

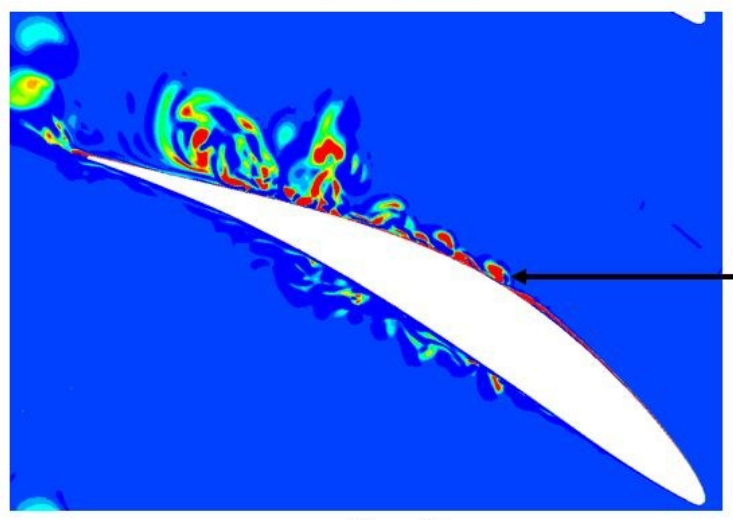

Baseline

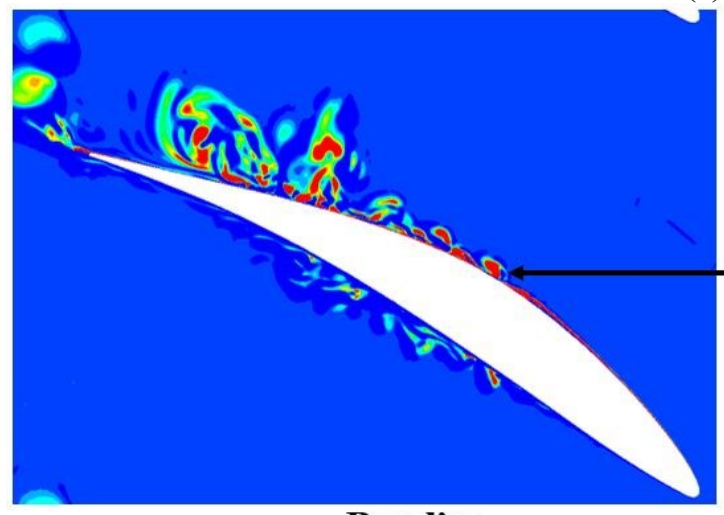

Baseline

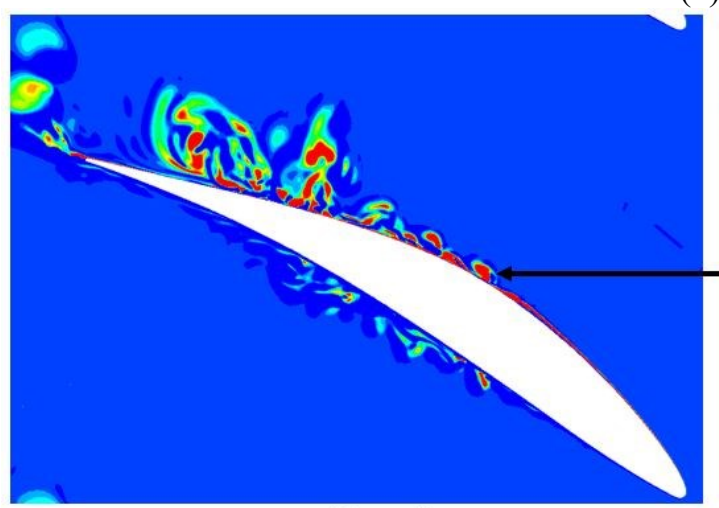

Baseline

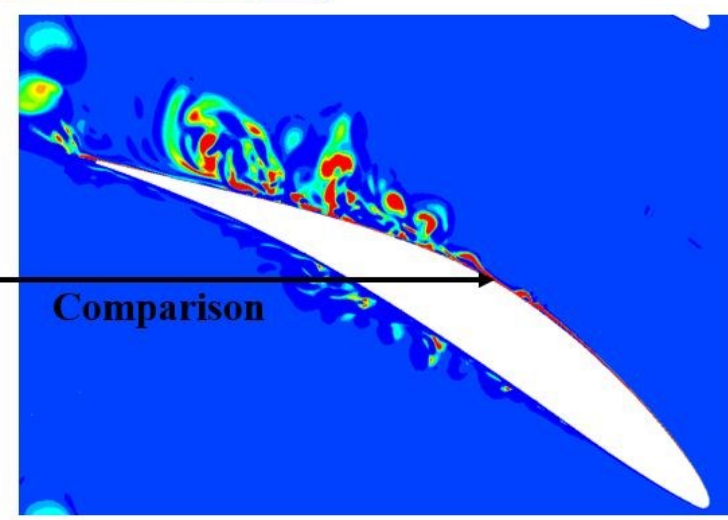

Actuation

(a) $4 \mathrm{~mJ}$

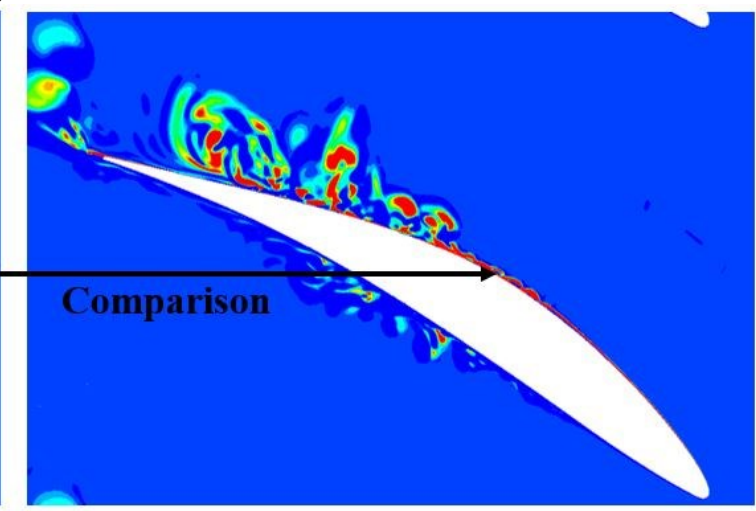

Actuation

(b) $2 \mathrm{~mJ}$

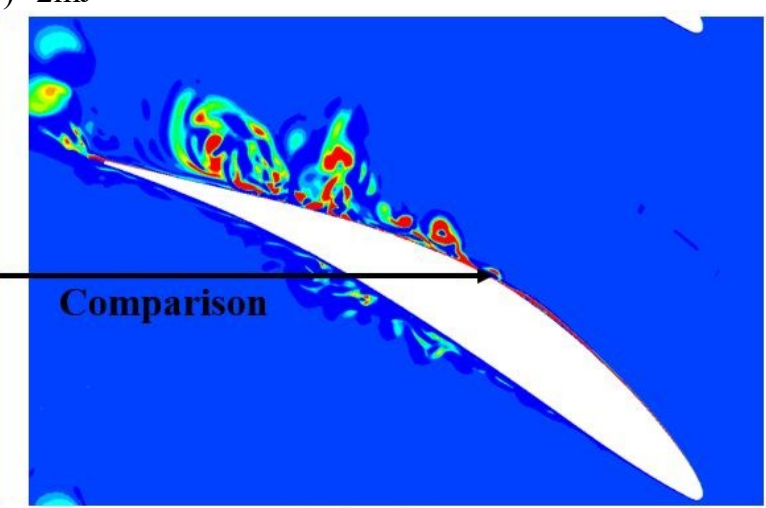

(c) $0.4 \mathrm{~mJ}$

\section{Actuation}

Fig. 13 The distributions of spanwise vorticity without and with plasma actuations (The energy input of plasma actuation is $4 \mathrm{~mJ}$, $2 \mathrm{~mJ}$ and $0.4 \mathrm{~mJ}$ repectively and the simulation timestep is 4000 ) 


\section{CONCLUSIONS}

The flow control effects of nanosecond plasma actuation on the boundary layer flow of a typical CDA compressor airfoil are investigated in this paper using LES simulation method, and main conclusions obtained are listed as below.

The boundary layer flow remains as laminar at the front part of the blade, and under the influences of laminar separation bubble, the boundary layer flow transits to turbulent at the rear part of the blade.

The plasma actuations located at the blade leading edge can induce a small spanwise vortex through influencing on the density and pressure of the flow field. As the small spanwise vortex moves downstream along the blade surface with the main flow, it can suppress the turbulent flow mixing and reduce the total pressure loss. The flow control effect of the small spanwise vortex is summarized as wall jet effect.. However, the small spanwise vortex induced by the plasma actuation will increase the total pressure loss of the laminar boundary layer flow while moving by. So, it is also beneficial to spare extra efforts to reduce the total pressure loss increase within the laminar boundary layer flow as the small vortex structure moves by.

\section{ACKOWLEDGEMENTS}

This paper is supported by the National Natural Science Foundation of China (Nos. 51790511).

\section{References}

T C Corke, C L Enloe, S P Wilkinson (2010). Dielectric barrier discharge plasma actuators for flow control. Annual Review of Fluid Mechanics 42(1): 505-529

H D Zhang, X J Yu, B J Liu, et al. (2017). Control of corner separation with plasma actuation in a high-speed compressor cascade. Applied Sciences 7(5): 465

H D Zhang, Y Wu, Y H Li, et al. (2019). Control of Compressor Tip Leakage Flow Using Plasma Actuation. Aerospace Science \& Technology 86(-): 244-255

E Akcayoz, H D Vo, A Mahallati (2016). Controlling corner stall separation with plasma actuators in a compressor cascade. Journal of Turbomachinery 138(8): 081008

D E Hobbs, H D Weingold (1984). Development of controlled diffusion airfoils for multistage compressor application. Journal of Engineering for Gas Turbines and Power 106(2): 271-278

M G De Giorgi, E Pescini, F Marra, et al. (2016). Plasma actuator scaling down to improve its energy conversion efficiency for active flow control in modern turbojet engines compressors. Applied Thermal Engineering 106(-): 334-350

J G Zheng, Y D Cui, B C Khoo (2019). A comparative study of alternating current and nanosecond plasma actuators in flow separation control. International Journal of Heat and Mass Transfer 135(-): 10971117

Y H Li, Y Wu, M Zhou, et al. (2010). Control of the corner separation in a compressor cascade by steady and unsteady plasma aerodynamic actuation. Experiments in Fluids 48(6): 1015-1023

J Kriegseis, B Simon, S Grundmann (2016). Towards InFlight Applications? A Review on Dielectric Barrier Discharge-Based Boundary-Layer Control Applied Mechanics Reviews 68(2): 020802

M N Goodhand (2011). Laminar flow compressor blades. In: Proceedings of the 9th Osborne Reynolds Colloquium and Research 2011, London

S Saddoughi, G Bennett, M Boespflug, et al. (2014). Experimental investigation of tip clearance flow in a transonic compressor with and without plasma actuators. Journal of Turbomachinery 137(4): 041008

S Traficante, M G De Giorgi, A Ficarella (2016). Flow Separation Control on a Compressor-Stator Cascade Using Plasma Actuators and Synthetic and Continuous Jets. Journal of Aerospace Engineering 29(3): 04015056

V Motta, L Malzacher, D Peitsch, et al. (2018). A Physically Consistent Reduced Order Model for Plasma Aeroelastic Control on Compressor Blades. In: Proceedings of the ASME. Turbo Expo: Power for Land, Sea, and Air 2018, Oslo, Norway,

D V Ness, T C Corke, S C Morris (2012). Plasma actuator blade tip clearance flow control in a linear turbine cascade. Journal of Propulsion \& Power 28(3): 504516

H D Vo (2010). Rotating stall suppression in axial compressors with casing plasma actuation. Journal of Propulsion \& Power 26(4): 808-818

H D Vo, J D Cameron, S C Morris (2008). Control of short length-scale rotating stall inception on a high-speed axial compressor with plasma actuation. In $A S M E$ : ASME.

A R Wadia, G Jothiprasad, S Saddoughi (2012). The effect of plasma actuators on tip clearance flows in compressor rotors. In: Proceedings of the ICAS 2012-28 ${ }^{\text {th }}$ International Congress of the Aeronautical sciences 2012, Brisbane, Australia

J Y Yu, J N Yu, F Chen, et al. (2019). Numerical study of tip leakage flow control in turbine cascades using the DBD plasma model improved by the parameter identification method. Aerospace Science and Technology 84(-): 856-864

Aleksandrov N. L., Kindysheva S. V., Nudnova M. M., et al. (2010). Mechanism of ultra-fast heating in a nonequilibrium weakly ionized air discharge plasma in high electric fields. Journal of Physics D: Applied Physics 43: 255201

Starikovskii A. Y., Nikipelov A. A., Nudnova M. M., et al. (2009). SDBD plasma actuator with nanosecond pulse-periodic discharge. Plasma Sources Science And Technology 18: 034015

Steinert W., Eisenberg B., Starken H. (1990). Design and Testing of a Controlled Diffusion Airfoil Cascade for Industrial Axial Flow Compressor Application. In: Proceedings of the Gas Turbine and Aeroengine Congress and Exposition 1990, ASME, Brussels, Belgium 
Steinert W., Starken H. (1996). Off-Design Transition and Separation Behavior of a CDA Cascade Journal of Turbomachinery 118(2): 204-210

Zhao G. Y., Li Y.H., Liang H., et al. (2015). Phenomenologicalmodelingofnanosecondpulsedsurf acedielectricbarrierdischargeplasmaactuation for flow control Acta Physica Sinica 64: 015101 